Józef Młyński

Cardinal Stefan Wyszynski University in Warsaw, Poland

\title{
Sozialarbeit als zielgerichtetes Handeln
}

\author{
Social Work as a Purposeful Action
}

\section{Abstract}

Social work since the beginning of the history of science has been interesting for many representatives of various scientific disciplines. It is so, because both the subject and object of its activity is man - an individual who does not always cope with proper functioning in his own environment and society. In this context, social work is a purposeful activity in favour of an individual, a group, a family, society. Social work possesses a wide spectrum of fields and as a purposeful and professional activity is not only needed, but also necessary for supporting a human being.

\section{Keywords}

Social work, a purposeful activity, an individual, society.

\section{Einleitung}

In der Gesellschaft und im Berufsleben macht sich jeder Mensch Gedanken über die Wirklichkeit, die ihn umgibt. Er bemerkt in ihr viele Phänomene: gesellschaftliche Stratifikation und Segregation; verschiedene Kulturformen; das ganze Ensemble von Faktoren, die verantwortlich sind für das Ethos menschlichen Verhaltens und die mehr oder weniger das menschliche Bewusstsein prägen. Diese Wirklichkeit kann von zwei verschiedenen Standpunkten aus geprüft werden. Der erste umfasst den Wandel, der in der mikrostrukturellen Welt stattfindet, der zweite dagegen globale Phänomene, die zur Welt der Makrostrukturen gezählt werden. Sowohl auf der ersten als auch der zweiten Ebene der Analyse der Wirklichkeit nimmt das menschliche Individuum einen wichtigen Platz ein. Oft 
erfährt der Mensch jedoch in der von globalem Wandel und Pluralismus geprägten Gesellschaft ein Gefühl des Verlorenseins. Auf der einen Seiten faszinieren ihn der gesellschaftliche Wandel, die ganze Wirklichkeit, auf der anderen Seite jedoch kann er sich nicht mit anderen Leuten verständigen, auch wenn sie am gleichen Ort wohnen. Die interpersonellen Beziehungen unterliegen Störungen auf verschiedenen Ebenen der menschlichen Koexistenz ${ }^{1}$.

$\mathrm{Zu}$ den vielen Ursachen, die man für die Disfunktion des Menschen in der heutigen Welt aufzählen kann, gehören: gesellschaftlicher Wandel, Wertekrise, gesellschaftliche Entfremdung, Deprivation, verschiedene Formen des Verlorenseins, wie auch Störungen interpersoneller Beziehungen. In einer solchen Wirklichkeit benötigt der Mensch Hilfe. Es existieren verschiedene Bereiche von Hilfe, unter denen die Sozialarbeit den ersten Platz einnimmt. Das ist nicht verwunderlich, denn sie ist zielgerichtetes Handeln, gerichtet auf ein konkretes Individuum, eine Gruppe oder eine Organisation. Von ihrem breiten beruflichen Spektrum zeugt eine Reihe von Phänomenen, bei denen sich die Sozialarbeit als professionelles Hilfsinstrument erweist. In diesem Kontext soll in der folgenden Arbeit eine Analyse der Definitionen von Sozialarbeit und ihrem zielgerichteten Handeln erfolgen.

\section{Sozialarbeit - Begriffsdefinition}

Die Definition von Sozialarbeit ist ein schwieriges Unterfangen. Auf der einen Seite assoziieren wir sie mit Sozialhilfe und auf der anderen Seite ist sie mehr; daher auch die Mehrdeutigkeit des Begriffs. Zudem ist Sozialarbeit eine zu junge Disziplin, zumindest auf dem Markt der Wissenschaftszweige in Polen, um sie eindeutig zu definieren. Dem allgemeinen Verständnis nach ist sie berufliches Handeln mit dem Ziel, dem Individuum in der Gesellschaft dabei zu helfen, besser zu leben oder ihm die Fähigkeit zurückzugeben, besser innerhalb der Gesellschaft zu funktionieren.

Es existieren einige Definition von Sozialarbeit. Unter den vielen Varianten lohnt es sich, der Definition von H. Radlińska Beachtung zu schenken. Ihrer Meinung nach besteht Sozialarbeit aus dem Hervorbringen und Vergrößern menschlicher Kräfte, aus ihrer Verbesserung und der Organisation gemeinsamen Handelns für das Wohl der Menschen². Offizielle Dokumente der National Organisation

${ }^{1}$ Vgl. J. Młyński, Człowiek wobec pomocy... Zarys pracy socjalnej, Tarnów 2009, Verlag Biblos, S. 9.

${ }^{2}$ Vgl. H. Radlińska, Pedagogika społeczna, Warszawa 1961, Verlag Żak, S. 354. 
of Social Workers (NASW) stellen fest, dass Sozialarbeit die professionelle Fähigkeit ist, dem Individuum und gesellschaftlichen Gruppen Hilfe zukommen zu lassen bei der Bereicherung oder dem Aufbau ihrer Fähigkeiten, innerhalb der Gesellschaft zu funktionieren, wie auch gesellschaftliche Bedingungen zu schaffen, die diesem Ziel dienen. Die Praxis der Sozialarbeit stützt sich auf professionelle Einarbeitung - in die Werte und Techniken mit dem Ziel, eines der folgenden Resultate zu erreichen: Menschen mit gezielten Hilfestellungen zu unterstützen; mit Ratschlägen und Psychotherapie einem Individuum, einer Familie oder einer Gruppe zu dienen; Gemeinschaften oder Einzelnen bei der Einführung oder Verbesserung sozialer und medizinischer Dienste zu helfen und sie auch an den zugehörigen legislativen Prozessen teilhaben zu lassen. Die Praxis der Sozialarbeit erfordert Wissen über menschliche Entwicklung und menschliches Verhalten, über gesellschaftliche, ökonomische und kulturelle Institutionen und über Wechselbeziehungen zwischen diesen Faktoren ${ }^{3}$.

B. Du Bois und K. Krogsrud Miley bezeichnen Sozialarbeit als professionelles Handeln, das sowohl die Verbesserung der Lebensbedingungen des Individuums und der Gemeinschaft, als auch die Linderung menschlichen Leidens und die Lösung gesellschaftlicher Probleme zum Ziel hat ${ }^{4}$. Sozialarbeiter werden folglich als berufliche Betreuer bezeichnet, die mit den Menschen arbeiten, um ihre Fähigkeiten zu entwickeln und ihre Handlungsmöglichkeiten zu vergrößern, soziale Hilfe und Mittel zur Verfügung zu stellen, soziale Dienste zu organisieren, die menschlich und einfühlsam agieren, und gesellschaftliche Strukturen zu entwickeln, die die Möglichkeit zu solchem Handeln schaffen ${ }^{5}$. R. A. Skidmore und M. G. Thackeray stellen fest, dass man die Semantik des behandelten Begriffs als Kunst, Lehre und Beruf begreifen kann, die den Menschen bei der Lösung ihrer persönlichen Probleme wie auch ihrer Probleme innerhalb einer Gruppe (insbesondere der Familie) und Gemeinschaft derart hilft, dass auf diesen Ebenen zufriedenstellende Verhältnisse erreicht werden können ${ }^{6}$.

${ }^{3}$ Vgl. J. Szmagalski, Teoria pracy socjalnej a ideologia i polityka spoteczna, przykład amerykański, Warszawa 1994, Verlag Żak, S. 78.

${ }^{4}$ Vgl. B. Dubois, K. Krogsrud Miley, Praca socjalna, Warszawa 1996, Verlag BPS Śląsk, S. 25. S. 25.

${ }^{5}$ Vgl. B. Dubois, K. Krogsrud Miley, Praca socjalna, Warszawa 1996, Verlag BPS Śląsk,

${ }^{6}$ Vgl. R.A. Skidmore, M. G. Thackeray, Wprowadzenie do pracy socjalnej, Katowice 1998, Verlag BPS Śląsk, S. 23. 
In den Vereinigten Staaten, die der Geburtsort dieses Wissenschaftszweigs ${ }^{7}$ waren, hat die Sozialarbeit zum Ziel, das Funktionieren des Individuums in der Gesellschaft zu verbessern, sowohl individuell als auch innerhalb einer Gruppe, indem das Handeln auf die gesellschaftlichen Verhältnisse ausgerichtet wird und sich auf die Interaktion zwischen Mensch und Umgebung auswirkt ${ }^{8}$. In Deutschland, wo die Sozialarbeit nicht weniger entwickelt ist als in den Vereinigten Staaten, ist diese definiert als jegliche berufliche Tätigkeit im Dienst an den Menschen, die Unterstützung und Hilfe benötigen, umso mehr ist ihr Ziel, Individuen und Gruppen in die Lage zu versetzen, ihre Lebensführung möglichst selbstständig und selbstverantwortlich in Situationen gestalten zu können, in denen diese Aufgabe natürliche Gemeinschaften und gesellschaftliche Institutionen nicht erfüllen können?.

Aus der obigen Analyse ergibt sich, wie stark entwickelt der Begriff der Sozialarbeit ist, wie vielen Modifikationen er unterliegt. Zu Beginn war er verbunden mit sozialer Hilfe, dann mit sozialer Aktion, professionellem beruflichem Handeln, mit karitativer Tätigkeit. Als Schlussfolgerung muss man heute diesen Begriff als berufliches Handeln zur gesellschaftlich-erzieherischen Aktivierung sehen, deren Ziel der Ausgleich verschiedener Mängel ist, die

${ }^{7}$ Die Vereinigten Staaten betrachten sich als Mutterland der Sozialarbeit, obwohl auch andere Länder - u. a. Polen - ihren eigenen Beitrag zur Entwicklung dieses Gebietes geleistet haben. Auf dem amerikanischen Kontinent entstand die Sozialarbeit aus verschiedenen, spontan geschaffenen Formen der Selbsthilfe, die den Obdachlosen als eine Form von Sicherheit dienen sollten. Bis in die 30er Jahren des 20. Jahrhunderts halfen den amerikanischen Bürgern in schwierigen Lebenssituationen hauptsächlich die Institutionen und Organisationen, die auf dem Gebiet der Gemeinschaftshilfe tätig sind. Erst in den Jahren der Weltwirtschaftskrise entwickelten sich die Möglichkeiten der Sozialversicherung auf einer breiteren Skala. Vgl. B. Szatur-Jaworska, Teoretyczne podstawy pracy socjalnej, in: T. Pilch, I. Lepalczyk (Hrsg.), Pedagogika Społeczna, Warszawa 1995, Verlag Żak, S. 106. In Europa teilten sich die Bürger dagegen schon früher die Verantwortung für die soziale Sicherheit mit den Gemeinden (z. B. wurde das in Großbritannien im 1601 eingeführten Armengesetz, Act for the Relief of the Poor, geregelt), oder dem Staat (z. B. wurden in Deutschland in den 80er Jahren des 19. Jahrhunderts eine Reihe von Gesetzen verabschiedet, die die Grundlagen des Sozialversicherungssystems bilden). Die Vereinigten Staaten waren nicht nur ein Gebiet der dynamischen Entwicklung der praktischen sozialen Tätigkeit. Hier entstanden auch die ersten weithin bekannten theoretischen Arbeiten, die den Methoden der Sozialarbeit gewidmet waren. Klassische Werke aus diesem Bereich sind die Bücher von Mary Richmond, die eine soziale Diagnose (Social Diagnosis - 1917) und Methoden der sozialen Einzelfallhilfe behandeln.

${ }^{8}$ Vgl. R.A. Skidmore, A. Marciniak, Sozialarbeit. Sozialhilfe, in: Lexikon der pädagogik (Ergänzungsband), Freiburg 1964, Verlag Herder, S. 677; R.A. Skidmore, M.G. Thackeray, Wprowadzenie do pracy socjalnej, Katowice 1998, Verlag BPS Śląsk, S. 18.

${ }^{9}$ Vgl. A. Marciniak, Sozialarbeit. Sozialhilfe, in: Lexikon der pädagogik, Freiburg 1964, Verlag Herder, S. 677. 
verursacht werden durch fehlerhaftes Funktionieren des Individuums in der Gesellschaft.

\section{Bedarf an zielgerichteten Aktivitäten in der Sozialarbeit}

Dieselben Ideen und Traditionen, die Einfluss auf die Sozialpädagogik hatten, bildeten die eigentliche polnische Theorie der Sozialarbeit. Die unter dem Einfluss dieser Ideen entstandenen Einrichtungen der Erziehung und der Pflege, Bildungsinstitutionen und Verbände, die verschiedene gesellschaftliche Ziele verwirklichen, spielten in einer Gesellschaft, die immer wieder mit der Teilung des Staatsgebietes leben musste, in zweifacher Hinsicht eine Rolle. Auf der einen Seite kompensierten sie das Fehlen eines staatlichen Bildungssystems, staatlicher Erziehung, Kultur und sozialer Sicherheit. Auf der anderen Seite waren sie die Antwort der polnischen Gesellschaft auf die Phänomene Armut, kulturelle Rückständigkeit und fehlende Möglichkeit der freien Lebensgestaltung. Daher haben sich auch viele Aktive in ihrer Beurteilung der gesellschaftlichen Wirklichkeit auf die sozialistische Ideologie berufen. Besonderen Einfluss hatten in diesen Kreisen die Ansichten von L. Krzywicki und E. Abramowski ${ }^{10}$.

Sozialarbeit als zielgerichtetes Handeln ist verbunden mit der Realisierung praxeologischer und ethischer Grundsätze. Sie erfordert Respekt für das axiologische Spektrum gleichermaßen von denen, die professionell und beruflich Sozialarbeit verrichten, als auch von jenen, die sie ehrenamtlich ausüben. Die Entwicklung der ethischen Analyse der aus der axiologischen Ordnung stammenden Umstände, trat dann auf, als die Sozialarbeiter nach einer Stärkung ihrer beruflichen Tätigkeit gesucht haben, als sie begannen, nach der Verbesserung ihres beruflichen Status und Prestige zu streben. Sie waren nämlich überzeugt, dass die Sozialarbeit vor dem Hintergrund anderer, paramedizinischer und pädagogischer Berufe, tatsächlich als eigenständig erscheint ${ }^{11}$.

Die Wirkung der Sozialarbeit entwickelt sich recht stark und bietet viele Möglichkeiten, die mit der Wiederherstellung der den Individuen verloren gegangenen bzw. geschwächten Fähigkeiten zur individuellen oder kollektiven Existenz verbunden sind. Deshalb unterscheidet man einige wichtige Grundsätze der Ziele der Sozialarbeit. Zu den wesentlichen Zielen zählt: die Ausrichtung

${ }^{10}$ Vgl. B. Szatur-Jaworska, Teoretyczne podstawy pracy socjalnej, in: T. Pilch, I. Lepalczyk (Hrsg.), Pedagogika społeczna, Warszawa 1995, Verlag Żak, S. 115.

${ }^{11}$ Vgl. J. Młyński, Człowiek wobec pomocy... Zarys pracy socjalnej, Tarnów 2009, Verlag Biblos, S. 56. 
der Sozialarbeit auf das Lösen von Problemen, ihr Ziel ist die Befriedigung anerkannter menschlicher Bedürfnisse und Ambitionen; Sozialarbeit in der Einheit ihres Handelns lässt sich leiten von den Werten, die von der Gesellschaft festgelegt werden, die sowohl die Individuen (d. h. die Klienten) als auch die Sozialarbeiter verpflichten ${ }^{12}$. Das ist so, weil ihr grundlegendes Ziel die Verbesserung des gesellschaftlichen Funktionierens unabhängig davon ist, ob jenes Bedürfnis zur Verbesserung vom Individuum selbst bemerkt wird oder nur von der gesellschaftlichen Gesamtheit. Deshalb verfolgt nach Meinung von P. Pincus und A. Minahan die Sozialarbeit Ziele, die die Interaktion zwischen Menschen und ihrer gesellschaftlichen Umgebung betreffen, was wesentlichen Einfluss hat auf die Befriedigung grundlegender Lebensbedürfnisse des Menschen. Übereinstimmend mit dieser Überzeugung lassen sich für die Sozialarbeit vier grundlegende Ziele angeben:

- die Fähigkeit der Menschen zu festigen, Probleme zu lösen und mit ihnen zurechtzukommen,

- die Verbindung zwischen Menschen und Institutionen herzustellen, die die Mittel, Leistungen und Möglichkeiten zur Selbsthilfe bieten können,

- effektives und humanitäres Handeln dieser Institutionen zu fördern,

- einen Beitrag zur Ausarbeitung und Verbesserung der Sozialpolitik zu leisten ${ }^{13}$.

In der Methodik der Sozialarbeit spricht man von zielgerichtetem Handeln der Sozialarbeiter, denn als Berufstätige beziehen sie jenes Handeln auf konkrete Individuen. Jedoch existieren drei Methoden, Gruppen des Handelns, nämlich: soziale Einzelfallhilfe, Sozialarbeit mit Gruppen und Sozialarbeit mit Gemeinschaften. Jeder dieser Fälle besitzt eine besondere Eigenart, stets jedoch ist die Aufgabe zu helfen, also zu handeln in Richtung der Linderung eines Problems, der Lösung von Schwierigkeiten im konkreten Fall. Zu diesem Ziel bedient sich die Sozialarbeit eines Wissensspektrums, das aus vielen Wissenschaftszweigen stammt. Man darf nicht vergessen, dass Sozialarbeit einen interdisziplinären Charakter hat. Die Sozialarbeiter lassen sich leiten von Wissen aus den Gebieten Soziologie, Psychologie, Psychiatrie, Ethik, Medizin, Beratung, ...

Darüber hinaus lassen sich die Sozialarbeiter in ihrem zielgerichteten Handeln von zwei Modellen leiten, und zwar vom medizinischen Modell und vom Modell des methodischen Handelns. Das erste ist eine Hilfe für ihr

${ }^{12}$ Vgl. E. Mazurkiewicz, Sprawność działania socjalnego w pedagogice społecznej Heleny Radlińskiej, Wrocław 1983, Verlag WNW, S. 41.

${ }^{13}$ Vgl. R. A. Skidmore, M. G. Thackeray, Wprowadzenie do pracy socjalnej, Katowice 1998, Verlag BPS Śląsk, S. 21-22. 
Handeln, also unterscheidet es sich wesentlich vom zweiten. Deshalb wird es auch als Modell bezeichnet, als bestimmtes modellhaftes Beispiel zur Aufnahme zielgerichteten Handelns durch den Sozialarbeiter. In diesem Sinne beziehen sich die Sozialarbeiter auch auf die Phasen der Methodik. Es existieren zwei solche Phasen, und zwar die logische und die chronologische Ordnung. Ihr Unterschied hängt vom konkreten zielgerichteten Handeln des Sozialarbeiters ab.

Beim zielgerichteten Handeln des Sozialarbeiters muss man auch die Art und Weise des Handelns beachten. Es gibt bekanntlich ein breites Spektrum von Hilfe für ein Individuum oder eine Gruppe. Deshalb unterscheidet man auch eine gewisse Ordnung, und zwar die Analyse der Situation, die Bewertung, die Vereinbarung und die Beendigung. Man muss zu dem so konstruierten Verlauf der Ereignisse das mittelbare und das unmittelbare Handeln hinzufügen.

Sozialarbeit ist ohne jeden Zweifel deshalb zielgerichtetes Handeln, weil sie jeweils ein konkretes Subjekt betrifft. Das Subjekt ihres Handelns aber ist der Mensch. Es geht dabei um professionelles Handeln, für das entsprechendes Personal ausgebildet wird. Eine Eigenart des Berufs des Sozialarbeiters wird impliziert durch die Interaktionen zwischen Menschen und der gesellschaftlichen Umgebung, die Einfluss haben auf die Fähigkeit, Lebensaufgaben zu bewältigen, Unannehmlichkeiten zu verringern, Werte, Ambitionen und Lebensziele umzusetzen. Deshalb ist dieser Beruf eine praktische und wissenschaftliche Tätigkeit, zugleich auch die Kunst des Helfens. Unter den Instrumenten, derer sich die Sozialarbeit bedient, findet sich die Methode der Intervention, die dem Erreichen des grundlegenden Ziels dient, der Hilfe. Natürlich gibt es für den Gegenstand Sozialarbeit noch viele andere Ziele. In der polnischen Gesetzgebung werden diese Ziele formuliert als: Personen und Familien die Überwindung schwieriger Lebenssituationen ermöglichen, Leben unter Bedingungen, die der Würde des Menschen entsprechen, Unterstützung bei Anstrengungen, die Erfüllung unerlässlicher Bedürfnisse anstreben, selbstständige Lebensführung für Personen und Familien, wie auch ihre Integration in der Umwelt ${ }^{14}$.

Das theoretische Ziel der Sozialarbeit wird bestimmt als Hilfe für Personen und Familien zur Stärkung oder Wiedererlangung der Fähigkeit, in der Gesellschaft durch die Ausfüllung entsprechender gesellschaftlicher Rollen zu funktionieren, sowie der Schaffung von Bedingungen, die diesem Ziel förderlich sind ${ }^{15}$. Praktisch wird die Sozialarbeit jedoch klassifiziert in die beruflichen Kategorien der

${ }^{14} \mathrm{Vgl}$. A. Olech, Etos zawodowy prawników socjalnych, wartości, normy, dylematy etyczne, Katowice 2006, Verlag BPS Śląsk, S. 9.

${ }^{15}$ Art. 6, Ustawa o pomocy społecznej, 12.03.2004. 


\section{The Person and the Challenges \\ 216 Volume 1(2011) Number 2}

Hilfeleistenden, also in die, deren ontologische Dimension in der professionellen Gewährung von Unterstützung besteht ${ }^{16}$.

Das Ziel der Sozialarbeit wird auch erreicht über die Durchführung von Programmen zu Sozialhilfeleistungen für die Unterstützung oder den Aufbau günstiger Beziehungen zwischen Individuen und der Gesellschaft, um den Lebensstandard aller Bürger zu verbessern. Letztlich geht es darum, dass die Schützlinge selbstständig funktionieren können, also den Klienten die selbstständige Lösung von Problemen zu ermöglichen. Am allgemeinsten spiegeln vier komplementäre Ziele, die das Handeln im Bereich der Sozialarbeit beschreiben, das Ziel professionellen Handelns in diesem Beruf wider, das die Verbesserung der Daseinsbedingungen der Menschen und die Stärkung der Beziehungen zwischen Menschen und gesellschaftlichen Institutionen ist.

\section{Schlussfolgerung}

Die aufgezeigten Ziele erfüllen auf ihre Art den ideologisch-institutionellen Charakter des Funktionierens der Sozialarbeit, das sich direkt neben ihrem therapeutisch-unterstützenden Handeln platziert. Dieser Dualismus bedeutet, dass der heutige Sozialarbeiter auf der einen Seite ein professioneller psychologischpädagogischer Therapeut ist, auf der anderen aber die Funktion eines Beamten, eines Bürokraten als Repräsentant der öffentlichen Institutionen erfüllt. Dadurch unterliegen die Ziele, die man anstrebt und denen man sich verpflichtet, einem ständigen Wandel. Die Aktivität des Sozialarbeiters findet ihren Platz also zwischen den Grundsätzen der beruflichen Ethik und der gesellschaftlichen Hilfsinstitutionen, die im Rahmen der Umverteilungspolitik von Gemeinden, Städten und Ländern verfasst sind.

Ohne Zweifel tauchen im gesamten Prozess der Sozialarbeit verschiedene Arten von Schwierigkeiten auf. Solche Schwierigkeiten betreffen vor allem Menschen, denen Hilfe gewährt wird. Deshalb muss der Reichtum der Persönlichkeit des Sozialarbeiters in sich viele Merkmale enthalten, die notwendig sind zur Ausübung dieses Berufs: das strenge Befolgen von Grundsätzen der ausgeübten Arbeit und den guten Willen zur Umsetzung der nächsten, oft schwierigen Aufgaben.

${ }^{16}$ Das festgelegte Ziel der Sozialarbeit bestimmt dabei den Blickwinkel - und die eigentliche hauptsächliche Richtung und Motivation - auf die gesellschaftlichen Probleme, die Richtungen der Lösung zwischenmenschlicher Probleme, die Entscheidung strittiger Fragen, das Auftreten gegenüber dem bedürftigen Individuum. 
In diesem ausgezeichneten Bereich zielgerichteten Handelns, der Professionalität mit individuellen Eigenschaften verbindet, muss der Sozialarbeiter an den Wert guter Taten glauben und sich zur Ausübung dieses Berufes berufen fühlen. Er soll wissen, was von ihm erwartet wird und was er in einer bestimmten Situation tun muss, welche Methoden zur Hilfe er anwenden muss, wie er in seinem Handeln konsequent sein kann, dass er sich vom Wohl der Personen, denen er hilft, leiten lässt. Er muss ehrliche Zuneigung gegenüber den Menschen empfinden und ihre Probleme verstehen. Er darf keinen Zynismus empfinden, keine Boshaftigkeit und darf nicht zu vorschneller Kritik neigen. Das ist notwendig, damit er viel Geduld, Spontaneität und tröstliches Interesse hat, denn diese Eigenschaften tragen dazu bei, dass die Kontaktaufnahme einfach, natürlich und spontan ist.

Es wäre eine unzulässige Vereinfachung zu sagen, dass Sozialarbeit als zielgerichtetes Handeln sich auf ein bestimmtes Gebiet dieses Handelns beschränkt. Ihr Spektrum ist sehr weit und als zielgerichtete und professionelle Tätigkeit platziert sie sich am Fuße des Berges jeglicher Probleme des Menschen, der Hilfe benötigt und erwartet.

\section{Bibliography}

Art. 6, Ustawa o pomocy społecznej, 12.03.2004.

Dubois B., Krogsrud Miley K., Praca socjalna, Warszawa 1996, Verlag Żak.

Marciniak A., Sozialarbeit, Sozialhilfe, in: Lexikon der pädagogik, Freiburg 1964, Verlag Herder.

Mazurkiewicz E., Sprawność działania socjalnego w pedagogice społecznej Heleny Radlińskiej, Wrocław 1983, Verlag WNW.

Młyński J., Człowiek wobec pomocy... Zarys pracy socjalnej, Tarnów 2009, Verlag Biblos.

Olech A., Etos zawodowy pracowników socjalnych, wartości, normy, dylematy etyczne, Katowice 2006, Verlag BPS Śląsk.

Radlinska H., Pedagogika społeczna, Warszawa 1961, Verlag Żak.

Skidmore R.A., Marciniak A., Sozialarbeit. Sozialhilfe, in: Lexikon der pädagogik (Ergänzungsband), Freiburg 1964, Verlag Herder.

Skidmore R.A., Thackeray M.G., Wprowadzenie do pracy socjalnej, Katowice 1998, Verlag BPS Śląsk. 
Szatur-Jaworska B., Teoretyczne podstawy pracy socjalnej, in: T. Pilch, I. Lepalczyk (Hrsg.), Pedagogika społeczna, Warszawa 1995, Verlag Żak.

Szmagalski J., Teoria pracy socjalnej a ideologia i polityka społeczna, przykład amerykański, Warszawa 1994, Verlag Żak. 